\title{
Mental health and quality of life benefits of a pedometer-based walking intervention delivered in a primary care setting
}

\author{
Tomas Vetrovsky ${ }^{1, *}$, Jozef Cupka ${ }^{2}$, Martin Dudek ${ }^{3}$, Blanka Kuthanova ${ }^{4}$, Klaudia Vetrovska ${ }^{5}$, Vaclav \\ Capek $^{6}$, and Vaclav Bunc ${ }^{1}$ \\ ${ }^{1}$ Faculty of Physical Education and Sport, Charles University, Prague, Czech Republic; ${ }^{2}$ Mediciman s.r.o., Prague, Czech \\ Republic; ${ }^{3}$ Laureus s.r.o., Dobrichovice, Czech Republic; ${ }^{4}$ Praktici Praha 6, s.r.o., Prague, Czech Republic; ${ }^{5}$ Humilitas s.r.o., \\ Beroun, Czech Republic; and ${ }^{6}$ Second Faculty of Medicine, Charles University, Prague, Czech Republic
}

Copyright: (C) 2017 T. Vetrovsky et al. This is an open access article licensed under the Creative Commons Attribution License (http://creativecommons.org/licenses/by/4.0/).

\begin{abstract}
Background: Physical activity level is positively associated with mental health and health-related quality of life. Primary care providers are ideally situated to offer physical activity interventions, and pedometers are commonly used as motivational tools to increase walking. However, several recent trials of pedometer-based interventions in primary care settings neither improved patients' quality of life nor reduced anxiety or depression, but these interventions only had relatively modest effects on physical activity levels. Objective: Our aim was to assess whether a pedometer-based walking intervention delivered in a primary care setting affects anxiety, depression, and health-related quality of life. Methods: A quasi-experimental, pre-post, single group study was conducted in 23 physically inactive patients from four general practices who participated in a pedometer-based intervention. The patients were administered the Hospital Anxiety and Depression Scale (HADS) and MOS 36-Item Short-Form Health Survey (SF-36) questionnaires before and after the 3-month intervention. Results: Following the intervention, the patients increased their walking volume by 1,676 steps per day $(p<.001)$. Both the anxiety $(-1.4, p=.011)$ and depression $(-2.4, p=.001)$ subscales of HADS decreased, while the physical functioning $(+6, p=.023)$, social functioning $(+9, p=.035)$, mental health $(+12, p=.001)$, vitality $(+12, p=.003)$, and general health $(+7, p=.013)$ subscales of SF-36 increased. Conclusions: Providing physically inactive patients with a pedometer and encouraging them to walk more in a primary care setting was associated with lower anxiety and depression scores, and improved health-related quality of life.
\end{abstract}

Keywords: Hospital Anxiety and Depression Scale, MOS 36-Item Short-Form Health Survey, pedometer, physical activity, primary care, quality of life, walking

\section{Introduction}

Anxiety disorders and depression are the most frequently diagnosed psychological diseases, severely impacting the lives of the persons affected (Demyttenaere et al., 2004; Moussavi et al., 2007). Regular physical activity protects against the development of anxiety disorders and depression, reduces their symptoms, and increases the quality of life among patients with diagnosed anxiety disorders or depression (Cooney et al., 2013).

However, symptoms of anxiety and depression are common even among people without clinically

\footnotetext{
* Address for correspondence: Tomas Vetrovsky, Human Movement Laboratory, Faculty of Physical Education and Sport, Charles University, Jose Martiho 31, 16252 Prague 6, Czech Republic. E-mail: tomas.vetrovsky@gmail.com
}

diagnosed anxiety disorders or depression. Fortunately, physical activity seems to positively impact mental health and quality of life in these general, non-clinical populations, as well. Meta-analyses quantifying the effect of physical activity interventions on depression and anxiety in non-clinical populations found that physical activity reduces anxiety by a small effect and depression by a medium effect (Conn, 2010a, 2010b; Rebar et al., 2015). A small but significant effect of physical activity interventions on the psychological and physical domains of quality of life has also been reported in non-clinical populations (Gillison, Skevington, Sato, Standage, \& Evangelidou, 2009).

Primary care providers are ideally situated to offer physical activity interventions to these general populations. A meta-analysis found that promoting physical activity to sedentary adults in a primary care setting can 
significantly increase physical activity levels (Orrow, Kinmonth, Sanderson, \& Sutton, 2012). In addition, primary care providers themselves believe that they have a role in promoting physical activity among their patients (Hébert, Caughy, \& Shuval, 2012).

Although there are various types of physical activity, walking is one of the most effective; with little risk of injury among low-activity populations, it has been described as near perfect exercise (Morris \& Hardman, 1997). Compared with many sports, walking is a convenient and flexible form of exercise that can be incorporated into everyday life and sustained throughout a person's lifetime (Ogilvie et al., 2007). To increase the amount of walking, pedometers have been successfully employed as they serve as motivational instruments that provide feedback to patients (Bravata et al., 2007).

Surprisingly, little focus has been directed toward the benefits of pedometer-based walking interventions in primary care settings for mental health and quality of life in general, non-clinical populations. Most studies indicating that walking improves health-related quality of life utilize populations with chronic conditions (Conn, Hafdahl, \& Brown, 2009), and evidence from randomized controlled trials in general populations is limited (McMurdo et al., 2010; Mutrie et al., 2012). A meta-analysis of 8 walking-based clinical trials (Robertson, Robertson, Jepson, \& Maxwell, 2012), which found a large effect of walking on symptoms of depression, included adult participants who were already experiencing depression, and thus, it is uncertain whether its results can be generalized to non-clinical populations in primary care settings.

Recently, several large randomized controlled trials of pedometer-based walking interventions in primary care settings assessed anxiety and depression symptoms as secondary outcomes, but none of them showed significant effects on anxiety, depression, and healthrelated quality of life (Harris et al., 2017; Yates et al., 2017). Despite that, one group of authors indicated that during qualitative research conducted alongside the trial, most of their intervention participants verbally expressed that they were feeling better, sleeping better, had an improved mood, and had more energy and less pain (Normansell et al., 2014).

As a whole, the body of evidence on mental health and quality of life benefits of pedometer-based walking interventions in general, non-clinical populations in a primary care setting is contradictory and inconclusive. Therefore, our aim was to assess whether a pedometerbased walking intervention delivered in a primary care setting affects anxiety and depression symptoms and health-related quality of life in a general population of physically inactive adults without clinical anxiety disorders and depression.

\section{Methods}

A quasi-experimental, pre-post study was conducted in 4 general practices in the Czech Republic. The general practices were selected to represent various urban settings: 2 in a large city, 1 in a middle-sized town, and 1 in a small town. The protocol of the study was reviewed and approved by the Ethics committee of the Faculty of Physical Education and Sports, Charles University (081/2015).

Physically inactive adult patients were recruited during routine preventive visits with their general practitioner. Eligibility criteria for inclusion were: (1) less than 8,000 steps a day, determined objectively using accelerometers during a 7-day period; (2) age 18-64 years; and (3) written informed consent obtained before any assessment related to the study. Patients were excluded from participation on the following grounds (1) diagnosis of anxiety disorders or depression; (2) inability to walk for any reason; (3) co-morbid conditions that would affect adherence to the study procedures (e.g., inflammatory arthritis, active malignancy, renal disease requiring dialysis, cognitive impairment, or significant hearing or visual impairment); (4) pregnancy.

Before the intervention, all patients received a triaxial pedometer (eVito 3D Step Counter SL; HMM Diagnostics GmbH, Dossenheim, Germany) sealed with tape and were instructed to wear the pedometer every day and to not purposely increase their physical activity levels during the next week. After that, the average daily step count over the 7-day period was calculated and those patients who achieved more than 8,000 steps were excluded from the study. Patients without at least 4 valid days of measurement were also excluded. For the purpose of this study, a valid day was defined as having 8 or more hours of wear time.

Patients included in the study then participated in a 3-month physical activity intervention that consisted of a pedometer-based walking program with weekly step goals. The patients received an e-mail from a researcher, asking them to unseal the pedometer, wear it daily during their waking hours except when swimming or bathing, check the step count every evening, and gradually increase the daily number of steps to 10,000 . They were also required to upload data to a website at least once a week and encouraged to contact technical support if they experienced problems with data upload.

A subgroup of the patients also received additional e-mail-based counseling to help them achieve their step goal. A member of the research team, trained in behavioral techniques, sent them 8 e-mails based on behavioral principles, such as goal setting, self-monitoring, and personalized feedback. Specifically, the patients were assigned an individual progressive goal 
expressed as a weekly increase in the daily number of steps, determined as $15 \%$ of the subject's baseline value rounded to nearest hundred. Further, they were asked to suggest their own strategies to achieve this goal by identifying opportunities in their daily routine when they could include at least a 10-minute walk (e.g., park their cars farther away from a building's entrance, walk to/from lunch, walk before/after work). All e-mails were drafted individually and tailored to the specific needs of the participant and the circumstances of their case. However, the e-mails always incorporated some common features: (a) encouragement of the participants based on their achievement in the previous week, (b) reminder of the benefits of physical activity for the physical and mental health relevant to the individual participant, (c) discussion of individual behavioral strategies, (d) setting the goal for the upcoming week.

Before and after the intervention, patients were asked by a member of the research team to fill in two self-administered questionnaires to assess their mental health and health-related quality of life: the Hospital Anxiety and Depression Scale and the MOS 36-Item Short-Form Health Survey. The Hospital Anxiety and Depression Scale (HADS) is a 14-item questionnaire consisting of depression and anxiety subscales. The items are graded on a four-point Likert scale from 0-3 with the total score for each subscale ranging from 0-21 (Zigmond \& Snaith, 1983). The MOS 36-Item Short-Form Health Survey (SF-36) is a validated measure of health-related quality of life that consists of 36 questions divided into eight individually analyzed dimensions: (1) limitations in physical activities because of health problems (physical functioning); (2) limitations in usual role activities because of physical health problems (role-physical); (3) limitations in social activities because of physical or emotional problems (social functioning); (4) bodily pain; (5) general mental health; (6) limitations in usual role activities because of emotional problems (role-emotional); (7) vitality (energy and fatigue); and (8) general health perceptions. Each dimension is scored on a 0-100 scale with higher scores representing better self-reported health (Ware \& Sherbourne, 1992).

Data were tested for normality using the ShapiroWilk test. As their distribution was not normal, the differences between pre- and post-intervention scores were analyzed using the nonparametric Wilcoxon signed rank test. A $p$ value of $\leq .05$ was considered as statistically significant and all tests were two-tailed. Furthermore, two-sided $95 \%$ confidence intervals were constructed to describe the differences. All statistical analyses were performed using the statistical package R (version 3.3.3; R Foundation for Statistical Computing, Vienna, Austria).

\section{Results}

Twenty-three patients (12 males and 11 females) were included in the study with a mean age of 41 years ( \pm 10 ). With 13 obese and 5 overweight patients, the mean body mass index was $33 \mathrm{~kg} / \mathrm{m}^{2}( \pm 7)$. Their baseline physical activity during the 7-day period before the intervention was 5,043 steps per day $( \pm 1,377)$. There were no significant differences in baseline characteristics between male and female patients.

Post-intervention, the average daily step count over the 7-day period increased by 1,676 steps per day $(95 \%$ confidence interval: 783 to $2,569, p<.001$ ), which represents an increase of $33 \%$ from baseline. There were no significant differences between the subgroup that received the additional e-mail counseling and the subgroup that did not receive any additional support.

All patients filled out and returned the questionnaires both before and after the 3-month intervention, resulting in $100 \%$ of the patients being included in the analysis. At baseline, both the anxiety and the depression scores were lower than 7 , which is considered as a cut-off point for the presence of anxiety and depression. This is in line with the study eligibility criteria that excluded patients with a previous diagnosis of anxiety disorders or depression. Post-intervention, these scores further decreased as detailed in Table 1. Accordingly, the mental health subscale of SF-36 improved, as did four other subscales of SF-36: vitality, social functioning, physical functioning, and general health, as listed in Table 1.

\section{Discussion}

Our study shows that after a pedometer-based walking intervention delivered in a primary care setting, both mental health and health-related quality of life can be improved in a general, non-clinical population. The strengths of our study are the pragmatic design with recruitment from general practices, the proportional representation of both sexes, no dropouts or other losses for the entirety of the study duration, and a substantial increase in physical activity during the intervention in comparison with other walking interventions in primary care settings (Harris et al., 2017; Yates et al., 2017).

Our results are in line with those of previous metaanalyses of physical activity interventions in general populations that showed small but significant effects of physical activity on anxiety symptoms (Conn, 2010a; Rebar et al., 2015) and health-related quality of life (Gillison et al., 2009) in addition to small-to-medium effects on depression symptoms (Conn, 2010b; Rebar 
Table 1

Baseline and post-intervention values of the Hospital Anxiety and Depression Scale (HADS) and the MOS 36-Item Short-Form Health Survey (SF-36)

\begin{tabular}{lcccc}
\hline & $\begin{array}{c}\text { Baseline, } \\
\text { Mean }(S D)\end{array}$ & $\begin{array}{c}\text { Post-intervention, } \\
\text { Mean }(S D)\end{array}$ & $\begin{array}{c}\text { Change } \\
{[95 \% \mathrm{CI}]}\end{array}$ & $p$ value \\
\hline HADS & & & & \\
$\quad$ Anxiety & $6.6(3.3)$ & $5.2(2.3)$ & $-1.4[-2.4,-0.4]$ & .011 \\
$\quad$ Depression & $5.3(3.7)$ & $2.8(2.3)$ & $-2.4[-3.7,-1.2]$ & .001 \\
SF-36 & & & & \\
$\quad$ Physical functioning & $83(15)$ & $89(10)$ & $+6[+1,+11]$ & .023 \\
Role-physical & $78(34)$ & $77(34)$ & $-1[-20,+18]$ & .937 \\
Bodily pain & $86(21)$ & $81(23)$ & $-5[-18,+8]$ & .609 \\
General health & $59(16)$ & $66(15)$ & $+7[+2,+12]$ & .013 \\
Vitality & $51(17)$ & $63(18)$ & $+12[+5,+18]$ & .003 \\
Social functioning & $80(19)$ & $89(17)$ & $+9[+1,+17]$ & .035 \\
Role-emotional & $71(37)$ & $89(27)$ & $+17[-2,+36]$ & .120 \\
Mental health & $66(17)$ & $79(12)$ & $+12[+6,+19]$ & .001 \\
\hline
\end{tabular}

et al., 2015). Although these data agreed with ours, these meta-analyses included studies with a large variety of interventions, populations, and settings. As a result, it may be more appropriate to interpret our results in light of the more recent large trials of pedometer-based walking interventions in primary care settings (Harris et al., 2017; Yates et al., 2017). Surprisingly, none of these trials showed a significant effect of the intervention on anxiety, depression, or health-related quality of life compared to control groups.

This might be explained by a relatively modest effect of these trials' interventions on physical activity levels. While our intervention improved the daily step count by about $33 \%$, these trials only reported improvements ranging from $6 \%$ to $15 \%$. Interestingly, one older pedometer-based walking intervention in older adults succeeded to increase daily steps by $28 \%$ and improved the physical health (but not mental health) dimension score of the SF-36 questionnaire (Mutrie et al., 2012). Unfortunately, that study did not assess the anxiety and depression symptoms.

Another, though less probable, explanation of the confounding data between previous studies and the present could be the lower baseline anxiety and depression scores in the trial by Harris et al. (2017). While the baseline values in our study (anxiety 6.6, depression 5.3) are categorized as normal, the baseline values by Harris et al. (2017), who used the same HADS instrument, were markedly lower (anxiety 4.7, depression 3.9) and thus more difficult to improve by the end of the intervention. Unfortunately, Yates et al. (2017) also used the HADS instrument, but did not publish baseline values, meaning our data can only be directly compared to the data from Harris et al. (2017).

Although there were no dropouts within the present study and the intervention had a positive effect on physical activity as well as anxiety and depression symptoms, the low number of participants and especially the quasi-experimental design are limitations of the study and should be considered when interpreting our findings.

First, the quasi-experimental study cannot attribute the improvement in mental health and quality of life to the increase in physical activity. In fact, other factors like increased attention of the general practitioners and research staff, or increased self-awareness of patients due to repeated testing and regular self-monitoring might contribute to improved mental health and quality of life. However, as our aim was to test the effect of the intervention, not the causal role of the increased physical activity, our results are still valid with implications in primary care settings.

Second, the positive outcomes could be explained by natural improvement in anxiety and depression symptoms due to change of the season. Indeed, as our patients were recruited predominantly in winter months (from November to June), this might partially explain the increase in physical activity and improvement in depression scores, as the seasonal variations of physical activity (McCormack, Friedenreich, Shiell, Giles-Corti, \& Doyle-Baker, 2010; Shephard \& Aoyagi, 2009; Tucker \& Gilliland, 2007) and depression symptoms (Harmatz et al., 2000) are well documented. To examine this explanation, we tested whether the season of patients' recruitment was associated with their 
outcomes. Using the Kruskal-Wallis rank sum test, we did not find any association between the season of recruitment and the improvement in daily step count, anxiety and depression scores, and SF-36 subscales. Thus, we can effectively exclude that the improvements were due to the seasonal variations.

\section{Conclusion}

Our study showed that a pedometer-based walking intervention in a primary care setting with a positive effect on physical activity levels has the potential to improve mental health and health-related quality of life in a general population. However, due to limitations of the quasi-experimental design of our study and the fact that recent large randomized controlled trials have failed to display similar findings, this conclusion should be viewed with caution and should be verified in future large randomized controlled trials with mental health and quality of life measures as the primary outcomes.

\section{Acknowledgment}

We thank Tereza Veverkova for assistance in processing the questionnaires and data transcription. The study was funded in part by a research program of Charles University (Progres Q41).

\section{Conflict of interest}

There were no conflicts of interest.

\section{References}

Bravata, D. M., Smith-Spangler, C., Sundaram, V., Gienger, A. L., Lin, N., Lewis, R., ... Sirard, J. R. (2007). Using pedometers to increase physical activity and improve health: A systematic review. JAMA, 298, 2296-2304.

Conn, V. S. (2010a). Anxiety outcomes after physical activity interventions: Meta-analysis findings. Nursing Research, 59, 224-231.

Conn, V. S. (2010b). Depressive symptom outcomes of physical activity interventions: Meta-analysis findings. Annals of Behavioral Medicine, 39, 128-138.

Conn, V. S., Hafdahl, A. R., \& Brown, L. M. (2009). Metaanalysis of quality-of-life outcomes from physical activity interventions. Nursing Research, 58, 175-183.

Cooney, G. M., Dwan, K., Greig, C. A., Lawlor, D. A., Rimer, J., Waugh, F. R., ... Mead, G. (2013). Exercise for depression. Cochrane Database of Systematic Reviews, 2013, CD004366.
Demyttenaere, K., Bruffaerts, R., Posada-Villa, J., Gasquet, I., Kovess, V., Lepine, J. P., ... Chatterji, S. (2004). Prevalence, severity, and unmet need for treatment of mental disorders in the World Health Organization World Mental Health Surveys. JAMA, 291, 2581-2590.

Gillison, F. B., Skevington, S. M., Sato, A., Standage, M., \& Evangelidou, S. (2009). The effects of exercise interventions on quality of life in clinical and healthy populations; a meta-analysis. Social Science \& Medicine, 68, 1700-1710.

Harmatz, M. G., Well, A. D., Overtree, C. E., Kawamura, K. Y., Rosal, M., \& Ockene, I. S. (2000). Seasonal variation of depression and other moods: A longitudinal approach. Journal of Biological Rhythms, 15, 344-350.

Harris, T., Kerry, S. M., Limb, E. S., Victor, C. R., Iliffe, S., Ussher, M., ...Cook, D. G. (2017). Effect of a primary care walking intervention with and without nurse support on physical activity levels in 45- to 75-year-olds: The pedometer and consultation evaluation (PACE-UP) cluster randomised clinical trial. PLoS Medicine, 14, e1002210.

Hébert, E. T., Caughy, M. O., \& Shuval, K. (2012). Primary care providers' perceptions of physical activity counselling in a clinical setting: A systematic review. British Journal of Sports Medicine, 46, 625-631.

McCormack, G. R., Friedenreich, C., Shiell, A., Giles-Corti, B., \& Doyle-Baker, P. K. (2010). Sex- and age-specific seasonal variations in physical activity among adults. Journal of Epidemiology and Community Health, 64, 1010-1016.

McMurdo, M. E. T., Sugden, J., Argo, I., Boyle, P., Johnston, D. W., Sniehotta, F. F., \& Donnan, P. T. (2010). Do pedometers increase physical activity in sedentary older women? A randomized controlled trial. Journal of the American Geriatrics Society, 58, 2099-2106.

Morris, P. J. N., \& Hardman, A. E. (1997). Walking to health. Sports Medicine, 23, 306-332.

Moussavi, S., Chatterji, S., Verdes, E., Tandon, A., Patel, V., \& Ustun, B. (2007). Depression, chronic diseases, and decrements in health: Results from the World Health Surveys. Lancet, 370, 851-858.

Mutrie, N., Doolin, O., Fitzsimons, C. F., Grant, P. M., Granat, M., Grealy, M., ... Skelton, D. A. (2012). Increasing older adults' walking through primary care: Results of a pilot randomized controlled trial. Family Practice, 29, 633-642.

Normansell, R., Smith, J., Victor, C., Cook, D. G., Kerry, S., Iliffe, S., ... Harris, T. (2014). Numbers are not the whole story: A qualitative exploration of barriers and facilitators to increased physical activity in a primary care based walking intervention. BMC Public Health, 14, 1272.

Ogilvie, D., Foster, C. E., Rothnie, H., Cavill, N., Hamilton, V., Fitzsimons, C. F., \& Mutrie, N. (2007). Interventions to promote walking: Systematic review, BMJ, 334, 1204.

Orrow, G., Kinmonth, A.-L., Sanderson, S., \& Sutton, S. (2012). Effectiveness of physical activity promotion based in primary care: Systematic review and meta-analysis of randomised controlled trials. BMJ, 344, e1389.

Rebar, A. L., Stanton, R., Geard, D., Short, C., Duncan, M. J., \& Vandelanotte, C. (2015). A meta-meta-analysis of the effect of physical activity on depression and anxiety in non-clinical adult populations. Health Psychology Review, 9, 366-378. 
Robertson, R., Robertson, A., Jepson, R., \& Maxwell, M. (2012). Walking for depression or depressive symptoms: A systematic review and meta-analysis. Mental Health and Physical Activity, 5, 66-75.

Shephard, R. J., \& Aoyagi, Y. (2009). Seasonal variations in physical activity and implications for human health. European Journal of Applied Physiology, 107, 251-271.

Tucker, P., \& Gilliland, J. (2007). The effect of season and weather on physical activity: A systematic review. Public Health, 121, 909-922.
Ware, J. E., \& Sherbourne, C. D. (1992). The MOS 36-item short-form health survey (SF-36). I. Conceptual framework and item selection. Medical Care, 30, 473-483.

Yates, T., Edwardson, C. L., Henson, J., Gray, L. J., Ashra, N. B., Troughton, J., ... Davies, M. J. (2017). Walking away from type 2 diabetes: A cluster randomized controlled trial. Diabetic Medicine, 34, 698-707.

Zigmond, A. S., \& Snaith, R. P. (1983). The Hospital Anxiety and Depression Scale. Acta Psychiatrica Scandinavica, 67, 361-370. 